\title{
Accelerating the co-simulation method for the design of transmit array coils for MRI
}

\author{
Alireza Sadeghi-Tarakameh ${ }^{1,2}$ (D) Ehsan Kazemivalipour ${ }^{1,2} \cdot$ Umut Gundogdu $^{2} \cdot$ Serhat Erdogan $^{1} \cdot \operatorname{Ergin~}$ Atalar $^{1,2}$
}

Received: 25 January 2020 / Revised: 18 May 2020 / Accepted: 18 June 2020 / Published online: 27 June 2020

(c) European Society for Magnetic Resonance in Medicine and Biology (ESMRMB) 2020

\begin{abstract}
Objective Accelerating the co-simulation method for the design of transmit array (TxArray) coils is studied using equivalent circuit models.

Materials and methods Although the co-simulation method dramatically reduces the complexity of the design of TxArray coils, finding the optimum solution is not trivial since there exist many local minima in the optimization problem. We propose to utilize an equivalent circuit model of the TxArray coil to obtain a proper initial guess for the optimization process of the co-simulation method. To prove the concept, six different TxArray coils (i.e., three degenerate birdcage coils (DBC), two dual-row head coils, and one elliptical body TxArray coil) with two different loading strategies (cylindrical phantom and human head/body model) at $3 \mathrm{~T}$ field strength are investigated theoretically; as an example study, an eight-channel head-DBC is constructed using the obtained values.

Results This approach accelerates the design process more than 20 -fold for the coils that are investigated in this manuscript. Conclusion A fast and accurate method for tuning and decoupling of a TxArray coil can be achieved using its equivalent circuit model combined with the co-simulation method.
\end{abstract}

Keywords MRI $\cdot$ Transmit array $\cdot$ Co-simulation $\cdot$ Inductor calculations $\cdot$ Equivalent circuit model

\section{Introduction}

In this work, we introduce a fast method to find the optimum capacitor values for a transmit array (TxArray) coil.

There exist many benefits of using radiofrequency (RF) transmit array coils in magnetic resonance imaging (MRI). One common usage of this technology is for RF-shimming [1-4]. For the static magnetic field strength of $3 \mathrm{~T}$ or higher, the size of the human body becomes comparable to the wavelength at the Larmor frequency, and homogenous excitation cannot be achieved with the conventional birdcage

Electronic supplementary material The online version of this article (https://doi.org/10.1007/s10334-020-00858-0) contains supplementary material, which is available to authorized users.

Ergin Atalar

ergin.atalar@bilkent.edu.tr

1 Department of Electrical and Electronics Engineering, Bilkent University, 06800 Ankara, Turkey

2 National Magnetic Resonance Research Center (UMRAM), 06800 Ankara, Turkey body coil. Moreover, the $\mathrm{B}_{1}{ }^{+}$field is a function of patient position and size. An array of transmitters can be used to overcome these difficulties. Furthermore, in many RFshimming applications, the specific absorption rate (SAR) becomes a limiting constraint that can be taken under control by using TxArray coils [5-13]. Another possible use of the TxArray systems is to reduce the duration of the RF pulses designed for complex excitation patterns [14, 15]. As an example, the spatially selective excitation is used for limiting the size of the signal-contributing volume based on the region-of-interest (ROI), which is advantageous for fast data acquisition $[16,17]$. Another potential use for the TxArray systems is to achieve implant-friendly MRI scanning [18-22].

TxArray coils are studied in many forms, including decoupled strip-lines [23, 24], decoupled surface coils [25, 26], dipole-like structures [27-33] and degenerate birdcage coils (DBC) [34-37]. Despite the advantages of using TxArray coils for MRI, the design and manufacturing of such coils is a significant challenge due to the mutual coupling between elements of the array. One of the practical techniques described in the literature is constructing and tuning 
only one of the elements, initially, then assembling the remaining elements of the array in successive steps, followed by iteratively tuning and decoupling all elements at each step [34]. Unfortunately, this technique is very time-consuming, making it infeasible for a large number of elements.

The co-simulation method, which was proposed by Kozlov et al. [38], significantly reduced the difficulties of RF coil design methods. In this method, an electromagnetic (EM) simulation is performed by replacing all discrete elements (capacitors and inductors) with excitation ports. The calculated scattering matrix ( $S$ matrix) is exported to a circuit simulator in order to optimize the values of these elements. Using this method, the values necessary for tuning and decoupling can be accurately obtained. Additionally, it makes large problems (of many independent discrete elements) feasible to solve. However, in the optimization process of this method, the initial guess plays a significant role. In the literature $[39,40]$, many randomly chosen initial guesses were established to avoid converging to a local minimum, which makes the optimization process timeconsuming. On the other hand, there is no guarantee that the optimization will converge to the global minimum using the randomly chosen initial guesses. A pre-calculated initial guess was considered in an earlier study [9], but this strategy was not fully analyzed and did not yield the optimum solution in all cases that were tested.

In this work, we use analytic calculations and finite element method (FEM)-based simulations to obtain all inductive and resistive parameters necessary for the organization of the equivalent circuit model. Then, the circuit model is utilized in determining approximate values for the capacitors used in the design. These approximate values of capacitors are used as the initial values for the optimization process of the co-simulation method. To prove the effectiveness of the method, six different TxArray coils with two different loading schemes were investigated. The results of the proposed method are compared in terms of accuracy and speed to the results of the conventional co-simulation method with many randomly chosen initial guesses.

\section{Materials and methods}

Here, the proposed method will be explained in detail for an $\mathrm{N}$-channel shielded DBC shown in Fig. 1, while, the method can be easily extended for the other types of coils as well. In this design, there are three independent capacitor values: $C_{\mathrm{t}}$ is the tuning capacitor, $C_{\mathrm{d}}$ is the decoupling capacitor, and $C_{\mathrm{m}}$ is the matching capacitor when the load is a cylindrical uniform phantom.

In the N-channel DBC coil, there are $\mathrm{N}$ capacitors of each of the three types. Some capacitors, such as $C_{\mathrm{t}}$, can be distributed. The following theory aims to find an approximate

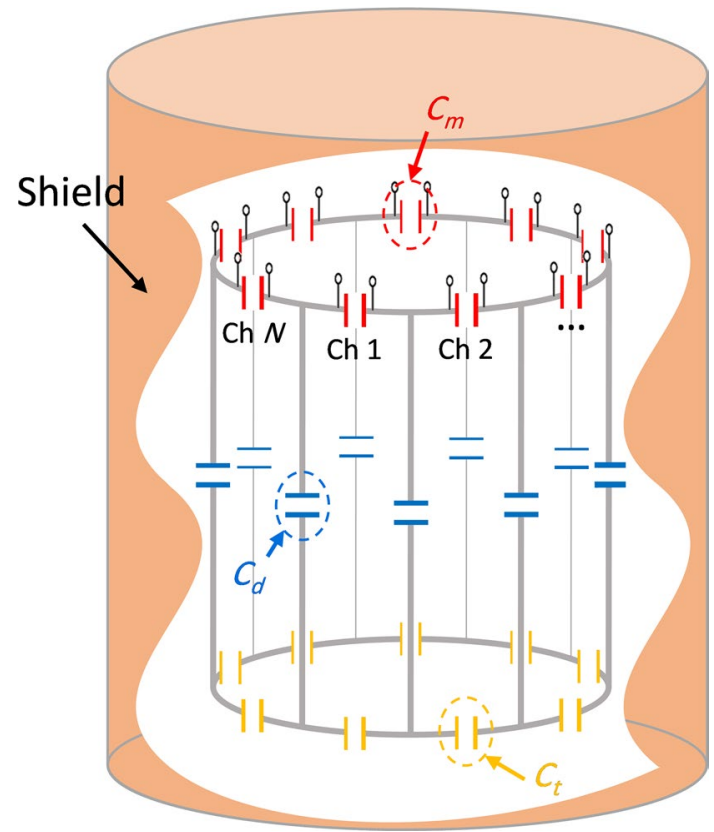

Fig. 1 An N-channel shielded degenerate birdcage coil. Tunning $\left(C_{\mathrm{t}}\right)$, decoupling $\left(C_{\mathrm{d}}\right)$, and matching $\left(C_{\mathrm{m}}\right)$ capacitors are shown as three independent capacitors in the design procedure

value for each of these capacitors and use them as initial guesses in the optimization process of the co-simulation technique. In the other designs or when the load is not circularly symmetric as in the human head/body model, the numbers of independent capacitor values can be significantly higher.

The flowchart of the proposed method is shown in Fig. 2. In this method, we obtain a coarse estimate for the decoupling and tuning capacitor values, based on the ideal decoupling between adjacent channels and assuming no loss in the system. Later, these values are used as the initial guesses for the optimization of a simplified circuit model of the array coil. The results of this intermediate optimization process are called the fine estimates. We used these as the initial guesses for the co-simulation method (see Fig. 2). These steps will be described below in detail.

\section{Equivalent circuit model}

First, we start with the equivalent circuit model of the coil. By modeling each wire or strip as an inductor-an assumption that is only valid if the wavelength is much larger than the strip segments-a shielded DBC can be modeled as an equivalent circuit that consists of self- and mutual-inductances. Assuming an infinitely long shield, the shield acts as an electromagnetic mirror. Therefore, the shielded DBC can be analyzed using the mirror currents. Finding the position of these mirror currents in a cylindrical coordinate system is a well-known procedure 


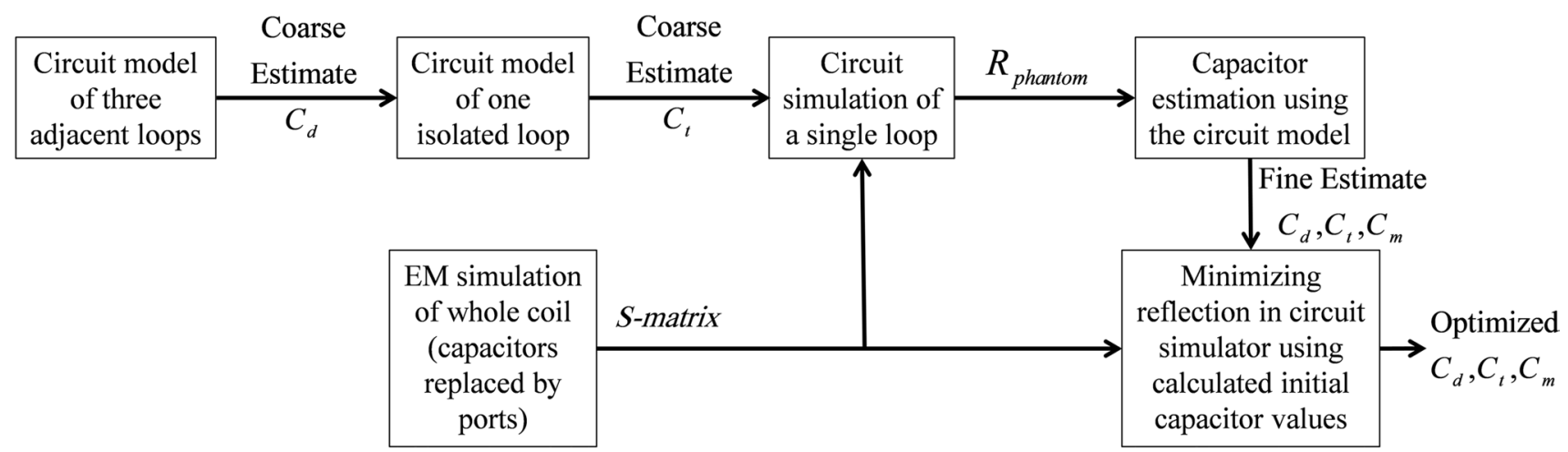

Fig. 2 The workflow of the proposed method

Fig. 3 a All combinations of self- and mutual-inductances in the DBC including the image of the coil corresponding to the shield. $\mathbf{b}$ The circuit model of three adjacent loops in a DBC was investigated for decoupling and tuning purposes. c Circuit model of a single loop, which was used to determine the loading effect on the coil. d The final equivalent circuit model of the DBC including the loading effect of the phantom
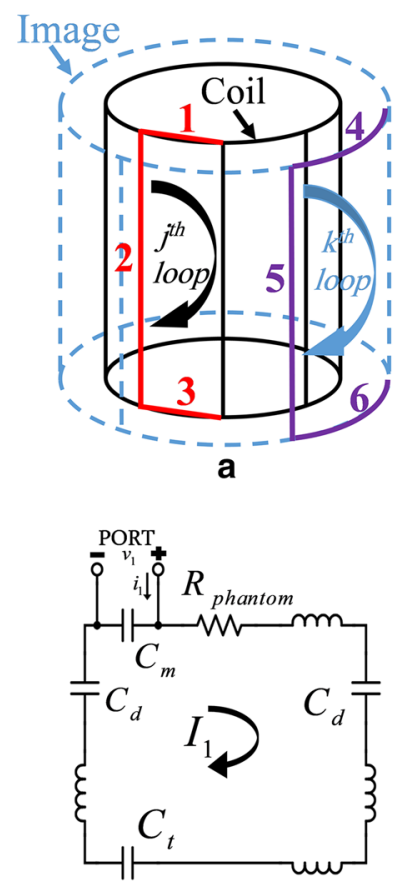

C

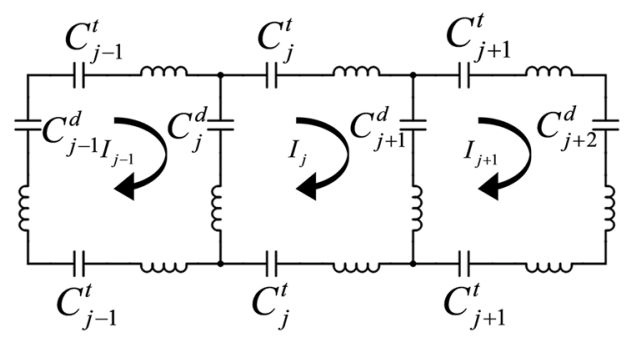

b

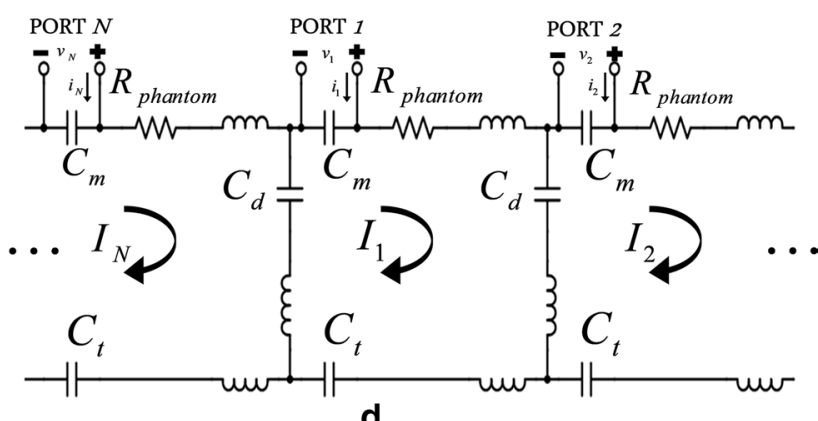

[41]. We designated the current segments on the coil 1 to 3 , and the mirror currents 4 to 6 , as shown in Fig. 3 a. Let $L_{j, p, k, q}$ represent the self- or mutual-inductance of the $p$ th segment in the $j$ th loop with the $q$ th segment in the $k$ th loop. Calculation of each of the inductance values can be found in the existing literature $[42,43]$. Kirchhoff's voltage law for the $j$ th loop can be written in the form of a matrix equation as follows [44]:

$\overline{\bar{K}} \cdot \bar{I}=\frac{1}{\omega^{2}} \overline{\bar{H}} \cdot \bar{I}$

where $\bar{I}$ is a vector of mesh currents, as shown in Fig. 3 b. This vector does not include the mirror currents since, by their definition, their values are identical to those of flow on the coil conductors. Therefore, the elements of matrices $\overline{\bar{K}}$ and $\overline{\bar{H}}$ can be written as [44]:

$$
\begin{aligned}
K_{j, k}= & 2\left(L_{j, 1, k, 1}-L_{j, 1, k, 3}-L_{j, 1, k, 4}+L_{j, 1, k, 6}\right) \\
& +\sum_{m=0}^{1} \sum_{n=0}^{1}(-1)^{m+n}\left[L_{j+m, 2, k+n, 2}-L_{j+m, 2, k+n, 5}\right]
\end{aligned}
$$

$H_{j, k}=2\left(\frac{1}{C_{t}}+\frac{1}{C_{d}}\right) \delta_{j, k}-\left(\frac{\delta_{j, k+1}}{C_{d}}+\frac{\delta_{j, k-1}}{C_{d}}\right)$

where $\delta_{j, k}$ is the Kronecker delta defined as: 
$\delta_{j, k}=\left\{\begin{array}{l}1 j=k \\ 0 j \neq k\end{array}\right.$

\section{Circuit model of three adjacent loops}

In the design of a DBC, decoupling between the channels, tuning to the desired frequency, and impedance matching of the input ports can be considered the essential parameters.

To find the decoupling capacitor value, we ignored the power loss of the phantom and assumed that the coupling between nonadjacent loops (channels) is negligible. Consequently, the coupling between adjacent loops with a common rung was canceled using an appropriate capacitor on the common rung. Figure $3 \mathrm{~b}$ shows the equivalent circuit model of three adjacent loops, such that the loop in the middle (jth loop) is supposed to be decoupled from the $(j-1)$ th and $(j+1)$ th loops. Therefore, one can obtain a coarse estimate for the decoupling capacitance as shown below.

$C_{d}=-\frac{1}{\omega^{2} K_{j, j-1}}$

\section{Circuit model of one isolated loop}

To find the tuning capacitor values, we assume perfect decoupling between all channels, no loss (as before), and that the ports are disconnected. Under these assumptions, the array structure can be treated as $N$ separated single loop coils. The Kirchhoff's voltage law equation for this single loop can simply be written as:

$j \omega\left[K_{j, j}-\frac{2}{\omega^{2}}\left(\frac{1}{C_{t}}+\frac{1}{C_{d}}\right)\right] I_{j}=0$

Substituting the value of $C_{\mathrm{d}}$ from Eqs. (5) into (6) and solving the equation for $C_{\mathrm{t}}$ provides a coarse estimate for the tuning capacitance as follows:

$C_{t}=\frac{2}{\omega^{2}}\left(\frac{1}{K_{j, j}+K_{j, j-1}+K_{j+1, j}}\right)$

\section{Electromagnetic (EM) simulations of the coil}

Similar to the original co-simulation method [38], all of the lumped elements on the coil that are used for tuning, decoupling, and matching purposes were replaced by $50 \Omega$ ports in an EM simulation environment. The iterative meshing process of the EM simulation was stopped if the greatest difference between the $S$-parameters of two successive iterations was less than a pre-determined value. Otherwise, the simulator proceeded with finer meshes [45]. Eventually, the $S$ matrix extracted from the EM simulator was imported to a circuit simulator for performing a single-loop circuit simulation as well as determining an optimum set of capacitors.

\section{Circuit simulation of a single loop}

To obtain a finer estimate of the tuning and decoupling capacitance values and determine an estimate for the matching capacitance, the loss (loading effect) is introduced. To predict the loss, a circuit simulation of a single loop was performed by assigning zero-capacitance (i.e., large impedance) to lumped elements of all the loops excluding the intended loop. The values obtained for $C_{\mathrm{d}}$ and $C_{\mathrm{t}}$ in Eqs. (5) and (7), respectively, were used in the single-loop simulation. Based on the circuit model in Fig. $3 \mathrm{c}, R_{\text {phantom }}$ was formulated as follows:

$R_{\text {phantom }}=\operatorname{real}\left\{\frac{j}{\omega C_{m}\left(j \omega C_{m} Z_{\text {simulation }}-1\right)}\right\}$

where $Z_{\text {simulation }}$ is the impedance at the port and is determined by the EM simulator.

\section{Circuit model of all $\mathbf{N}$ loops}

Once $R_{\text {phantom }}$ is determined, the general equivalent circuit model for the DBC, including the matching capacitor and loading effect of the phantom (Fig. 3d), can be utilized for design purposes. The impedance matrix corresponding to the circuit model in Fig. 3d can be formulated as follows:

$\overline{\bar{Z}}=-\frac{j}{\omega C_{m}}\left[\overline{\bar{I}}+\left(j \omega \overline{\bar{K}}-\frac{j}{\omega} \overline{\bar{H}}+R_{\text {phantom }} \overline{\bar{I}}\right)^{-1}\left(\frac{j}{\omega C_{m}} \overline{\bar{I}}\right)\right]$

where $\overline{\bar{I}}$ is the identity matrix. Then, the scattering matrix of an $N$-port network, with corresponding impedance matrix $\overline{\bar{Z}}$, can be determined as follows [46]:

$\overline{\bar{S}}=\left(\overline{\bar{Z}}+Z_{0} \overline{\bar{I}}\right)^{-1} \cdot\left(\overline{\bar{Z}}-Z_{0} \overline{\bar{I}}\right)$

where the matrix $Z_{0}$ represents the characteristic impedance of transmission lines $(50 \Omega)$.

Once the $\mathrm{S}$ parameters are calculated, the cost function can be evaluated as the total reflected power from the ports while one of the ports is stimulated by unit power: 
Cost $=\sum_{n=1}^{N}\left|S_{n 1}\right|^{2}$

We used the steepest-descent method [47, 48] to minimize the cost function. If the cost difference between two consecutive iterations is less than a predetermined value, the algorithm is terminated. The final capacitor values become the initial values for the co-simulation method.

After performing the EM simulation, the resultant $S$ matrix was exported to the circuit simulator. In the circuit simulator, each port was connected to its corresponding capacitor. A fine estimate for the capacitors was calculated using the equivalent circuit model introduced above. Utilizing the optimization tool of the circuit simulator (which uses the gradient optimization algorithm) with the minimum reflected power constraints and finely estimated capacitor values as the initial guesses, the proper capacitor values were obtained.

\section{Simulations and experiments}

To verify the proposed method, six different TxArray coils with two different loading schemes are designed (see Fig. 4) and simulated. One of these designs is constructed and tested with an MRI experiment.

\section{Comparison of the proposed method with the original co-simulation method}

An eight-channel head DBC (Coil 1 in Fig. 4), a 12-channel head DBC (Coil 2 in Fig. 4), a 16-channel cylindrical body DBC (Coil 3 in Fig. 4), a 16-channel dual-row head coil (Coil 4 in Fig. 4), a 16-channel shifted dual-row head coil (Coil 5 in Fig. 4), and a 16-channel elliptical body coil (Coil 6 in Fig. 4) were investigated for proof of the concept. Each structure was investigated in the presence of two different loading schemes, cylindrical phantom (first row in Fig. 4) and human head/body model (second row in Fig. 4), at $3 \mathrm{~T}$ field strength. HFSS (ANSYS, Canonsburg, PA, USA) and Microwave Office (AWR Corp., El Segundo, CA, USA) were used as EM and circuit simulators, respectively. All computations and simulations were performed on a workstation with two quad-core Intel(R) Xeon(R) X5472 processors with a $3 \mathrm{GHz}$ clock rate and $32 \mathrm{~GB}$ RAM.

\section{Coil construction}

The eight-channel DBC was constructed on a plexiglass cylinder (Fig. 5a) with a diameter of $293 \mathrm{~mm}$. The length of rungs was $250 \mathrm{~mm}$, and the width of copper strips was $15 \mathrm{~mm}$. The RF-shield of the coil was built on a larger plexiglass cylinder (380 $\mathrm{mm}$ diameter and $500 \mathrm{~mm}$ length) (Fig. 5b). Gradient-induced eddy currents were reduced by slitting the shield in 16 equally spaced locations along the $z$-direction and connecting them with $1 \mathrm{nF}$ capacitors [49, 50] at the positions facing the end-rings of the coil. We used eight coaxial cables with adjusted bazooka baluns on each cable to carry the RF power from amplifiers to the coil.

The constructed DBC was also used as a receiver by placing a transmit/receive (T/R) switch between the coil, transmit amplifiers, and receiver system. The T/R switch structure and circuit schematic are pictured in Fig. 5c, d, respectively. There are two series-connected PIN diodes; two DC blocking capacitors; an RF choke (RFC), and a $90^{\circ}$ low-pass $\pi$-type LC network [51]. The scanner provides DC control signals to change the states of the switch. The DC signals are connected to the board by the RFCs. In the transmit mode, the scanner turns on the PIN diodes with a $100 \mathrm{~mA}$ current. The receive port is isolated utilizing the high impedance created by the shorted $90^{\circ} \pi$-network,
Fig. 4 Comparison between the modified and original co-simulation method. Coil 1: eight-channel head DBC, Coil 2: 12-channel head DBC, Coil 3: 16-channel body DBC, Coil 4: 16-channel dual-row head coil, Coil 5: 16-channel shifted dual-row head coil, and Coil 6: 16-channel elliptical body coil. All structured were investigated in the presence of a cylindrical phantom (first row) as well as head/body model (second row)

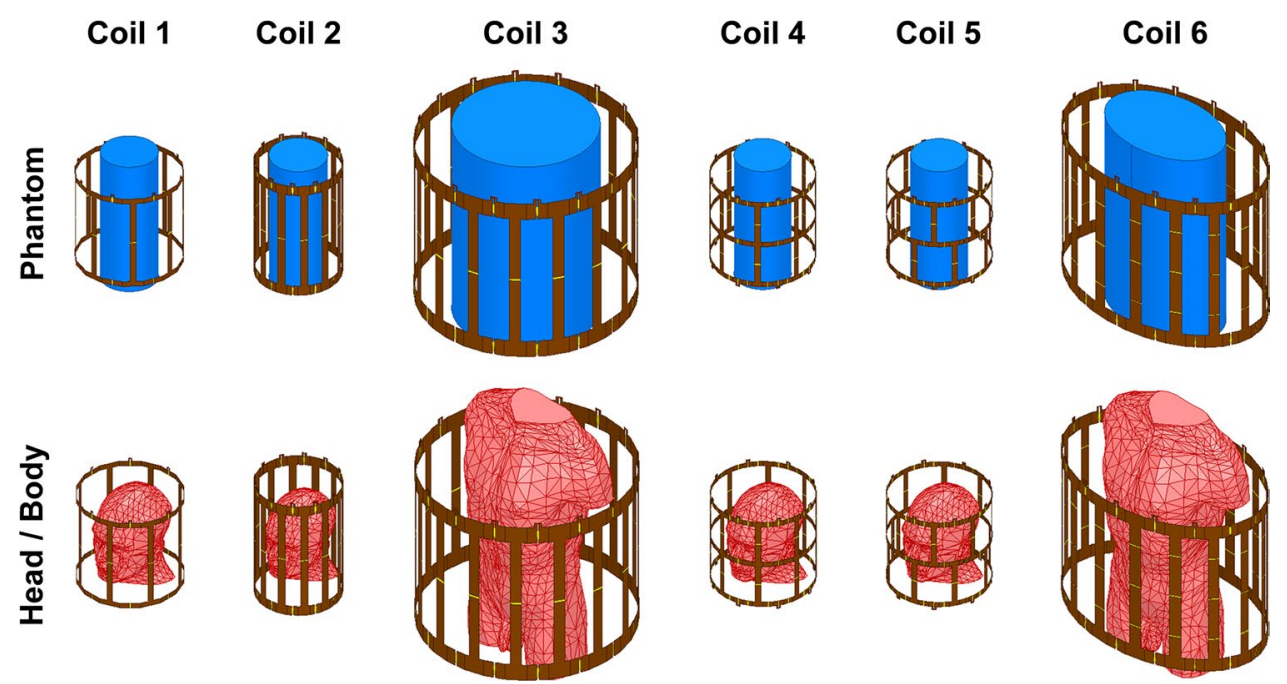



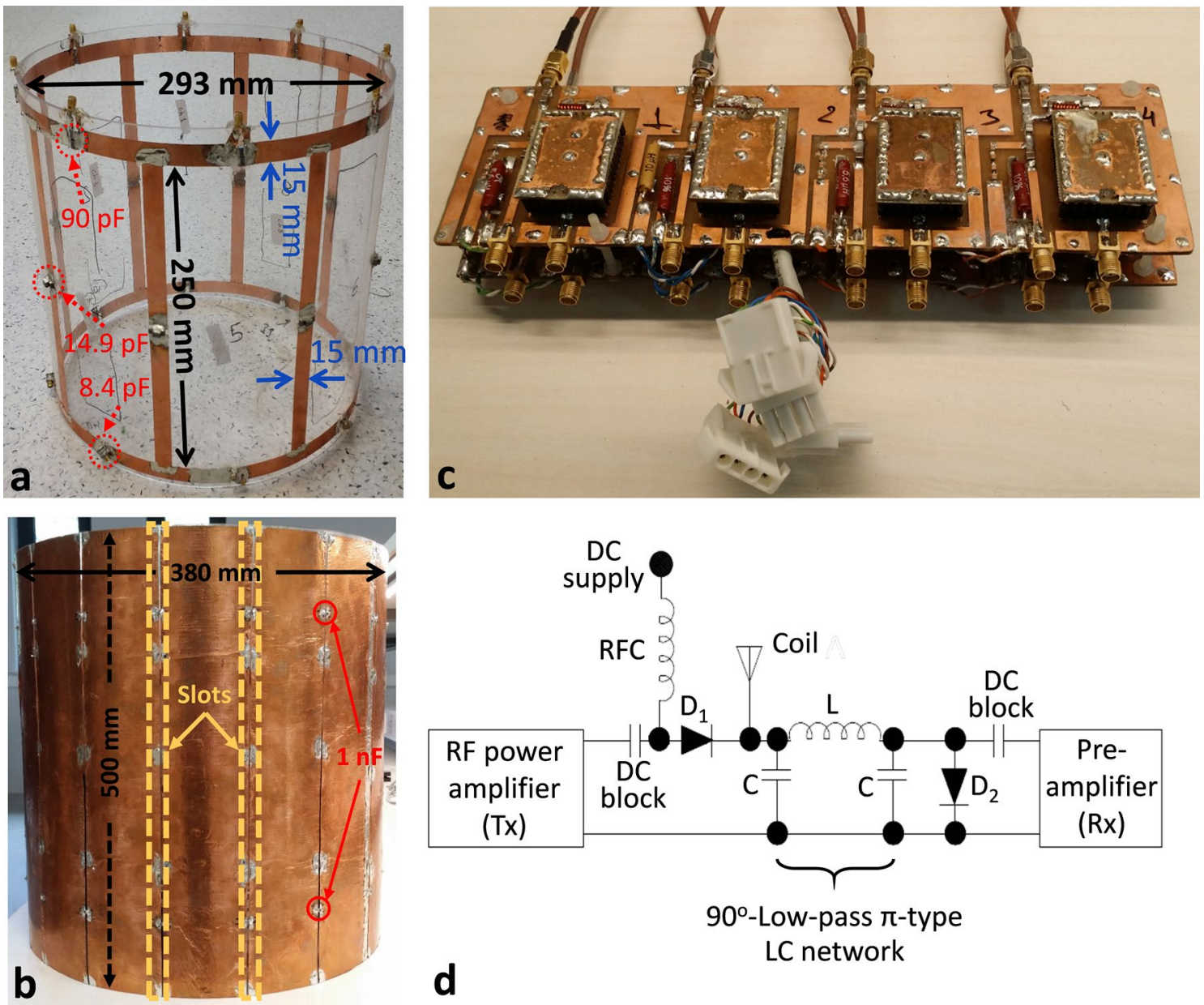

Fig. 5 Experimental setups. a An eight-channel DBC, which was designed and constructed using the proposed method. b An RFshield with several parallel slots in the $z$-direction on it (for reducing

which acts like a shorted quarter-wave transmission line. In the receive mode, the PIN diodes are turned off with a $-30 \mathrm{~V}$ signal so that the receiver port is connected to the coil over the low-pass LC network. The transmitting port is isolated using the high impedance created by the PIN diode, which is in the on-state. The isolation values are approximately $27 \mathrm{~dB}$ for both switching states, as determined by means of a bench-top measurement using a network analyzer (Agilent-E5061B, Baltimore, MD, United States).

\section{System configurations}

The parallel excitation was performed on a $3 \mathrm{~T}$ Tim Trio system (Siemens Medical Solutions, Erlangen, Germany) equipped with an 8-channel transmit array system. Each channel used a separate $8 \mathrm{~kW}$ RF amplifier (Analogic Corp., Boston, MA, USA). All of the experiments were performed

the eddy current effects); $\mathbf{c}$ The custom T/R switch used in the MRI experiment, $\mathbf{d}$ and the circuit model of the T/R switch

using a $150 \mathrm{~mm}$ diameter cylindrical SNR phantom (3.37 g/L NiCl $2.6 \mathrm{H}_{2} \mathrm{O}$ and $2.4 \mathrm{~g} / \mathrm{L} \mathrm{NaCl}$ ), which possessed electrical properties of $\varepsilon_{\mathrm{r}}=80$ and $\sigma=0.62 \mathrm{~S} / \mathrm{m}$ to mimic the human head. The conductivity of the phantom was measured using a magnetic resonance electrical properties tomography (MREPT) [52,53] experiment, and its relative permittivity was assumed to be the same as water.

\section{$B_{1}$ field mapping and sequence parameters}

$B_{1}^{+}$-maps were acquired using the Bloch-Siegert (BS) approach described by Sacolick et al. [54] A gradient-echo (GRE) pulse sequence was modified to apply Bloch-Siegert (BS) shift to spins by an off-resonance Fermi pulse. The duration and the off-resonance frequencies were selected to be $8 \mathrm{~ms}$ and $2 \mathrm{kHz}$, respectively. The other relevant imaging parameters were $\mathrm{TR}=100 \mathrm{~ms}, \mathrm{TE}=12 \mathrm{~ms}, \mathrm{NEX}=1$, $128 \times 128, \mathrm{FOV}=200 \mathrm{~mm}$. Each map was acquired individually in transmit mode, i.e., both slice selection and BS pulses 
were applied to each channel in transmission mode, one by one. To prevent unreliable data (low-SNR) from affecting the $\mathrm{B}_{1}$-maps, a mask with a threshold of one-tenth of the maximum $B_{1}$ value was implemented on the maps. Afterward, a quadrature interpolation was applied to the maps to smoothen them. All of the channels were used for reception, and a sum-of-squares method was used for image reconstruction. Acquired data were analyzed with the method described by Turk et al. [55]. The $B_{1}^{+}$relative phase maps were acquired by simply applying a GRE sequence with the same parameters, except for the Fermi pulse. The reference phase image was taken with the same GRE sequence when all channels were transmitting. The $B_{0}$ maps were acquired by a built-in field map sequence using the same imaging parameters, except with a $\mathrm{TE}_{1}$ of $5 \mathrm{~ms}$ and $\mathrm{TE}_{2}$ of $12.46 \mathrm{~ms}$.

\section{Results}

The performance improvement in the proposed modification to the co-simulation technique is demonstrated by comparing the original method to the modified technique.

\section{Comparison of the proposed method with the original co-simulation method}

As a reminder, in the original co-simulation technique, the capacitor values are found using an optimization technique, and the cost function (the total reflected power when unit input is provided from one of the channels of the DBC) is a nonlinear function of the capacitor values and has many local minima. Because of this difficulty, the optimization problem is solved by repeatedly using the steepest-descent method with different initial guesses and reporting the minimum of each solution as the global minimum, as described in the literature $[39,40]$.

In our proposed method, to find the capacitor values of the DBC, the equivalent circuit model was used as described in the previous section. In the case of the eightchannel head DBC shown in Fig. 5a, analytic calculations led to coarse estimates of $12.9 \mathrm{pF}$ and $24.5 \mathrm{pF}$ for $C_{\mathrm{d}}$ and $C_{\mathrm{t}}$, respectively. Then, an EM simulation of a single loop in the presence of the phantom was performed, and $R_{\text {phantom }}$ was determined to be $3.8 \Omega$. Afterward, an interstage optimization was performed to obtain a fine estimate for the three capacitor values. At this stage, $C_{\mathrm{d}}, C_{\mathrm{t}}$, and $C_{\mathrm{m}}$ were computed and found to be $13.9 \mathrm{pF}, 8.8 \mathrm{pF}$, and $89.5 \mathrm{pF}$, respectively. In the next step, all of the capacitors on the coil were replaced by $50 \Omega$ lumped ports in the simulation environment (Fig. 6a). The EM simulation was performed using the iterative meshing technique with $2.5 \times 10^{-3}$ stop criterion (i.e., the difference between the $\mathrm{S}$-parameters of two successive iterations) which led to 184,000 tetrahedral meshes in the last meshing step. After performing the EM simulation, the resultant $S$ matrix was exported to the circuit simulator. In the circuit simulator, each port was connected to the corresponding capacitor. Utilizing the optimization tool of the circuit simulator, which uses the gradient optimization algorithm, with the minimum reflected power constraints, the proper capacitor

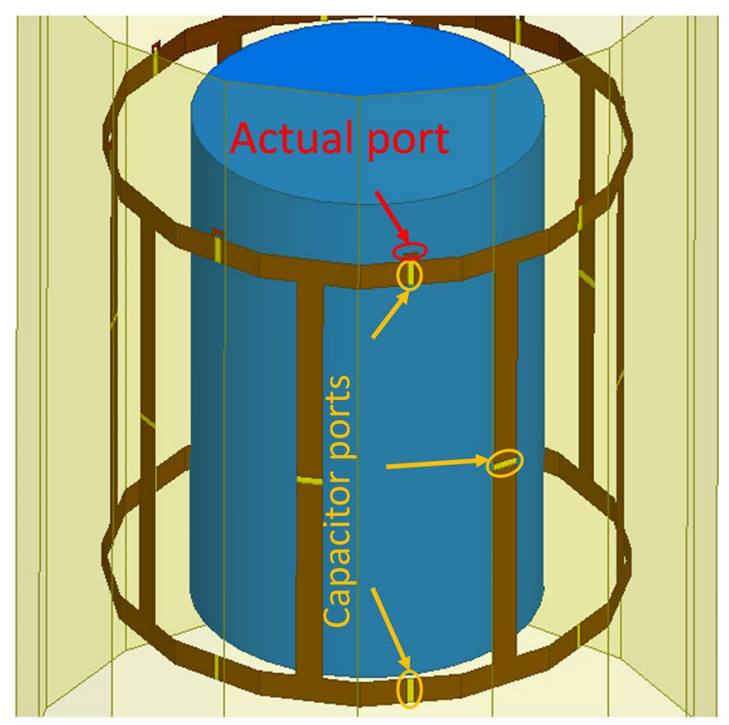

a

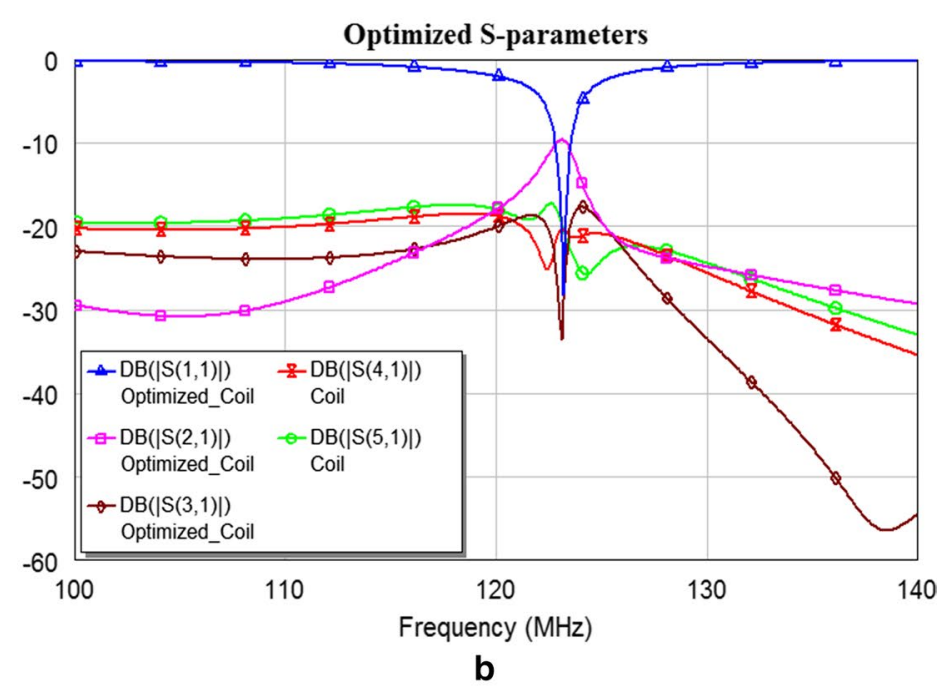

ports. b The optimized $S$-parameters using the circuit simulator with the constraint of minimum reflected power
Fig. 6 The procedure of the modified co-simulation method. a The EM simulation model of the DBC, including the phantom, used to extract the $S$-parameters when all capacitors were replaced with $50 \Omega$ 
values were obtained. The fine estimates for the capacitor values from the previous stage were used as the initial guesses for the optimization tool at the current stage. The optimized capacitor values were $14.9 \mathrm{pF}, 8.4 \mathrm{pF}$, and 90 $\mathrm{pF}$ for $C_{\mathrm{d}}, C_{\mathrm{t}}$, and $C_{\mathrm{m}}$, respectively. Figure $6 \mathrm{~b}$ shows the $S$-parameters obtained from the circuit simulator.

To compare the efficiency of the proposed method to the original co-simulation method, we repeated the original co-simulation design process as described in the literature [38-40].

All random values were chosen using the rand command in MATLAB (The MathWorks, Natick, MA, USA). By randomly choosing 1000 initial guesses (triple-sets of capacitor values in the range of $1 \mathrm{pF}$ to $100 \mathrm{pF}$ ), the optimization tool converged to various final cost values. Figure 7 a shows the converged cost values for different initial guesses. All circle markers correspond to the randomly chosen initial guesses, and the asterisk marker represents the cost value that was determined by considering the fine estimate for the initial capacitor values. This shows that finding the global minimum using randomly chosen initial guesses is not guaranteed, and it was achieved only three times by seeding with 1000 initial points. On the other hand, the global minimum was found in a single shot when the calculated initial values for the capacitors were used. The total reflected power from all ports for a given global minimum cost value was found by both methods to be 0.25 .
The duration of the optimization becomes an important factor for large problems. Figure $7 \mathrm{~b}$ demonstrates the number of iterations required for different initial guesses. The total number of iterations for 1000 randomly chosen initial guesses was over 794,000. However, the use of the calculated initial value provides the same results in 123 iterations. Thus, in this case study, the proposed algorithm speeds up the optimization process by more than 6400 times.

In Fig. 7c, the final cost values for various initial guesses were plotted with respect to the number of iterations. The cross marker, corresponding to the calculated initial values, provides the minimum cost value achieved using the fewest iterations.

To prove the concept, six different structures, shown in Fig. 4, with two different loading schemes, were investigated. The duration for each stage of the design for both methods is given in Table 1. Modified and original cosimulation methods were abbreviated as " $M$ " and " $O$ " in Table 1, respectively. Coils 1 through 6 refer to the structures shown in Fig. 4. The number of independent capacitors represents the number of independent variables that were optimized to achieve the minimum cost function.

In all cases, the number of initial capacitor values (seeds) was chosen to be 1000 to ensure that the global minimum would be achieved. This number is comparable to that used by Kozlov et al. [39, 40] (3000 seeds). For each of the initial capacitor values (seeds), the gradient optimization tool, with
Fig. 7 Analytically calculated initial value vs. randomly chosen initial values. a Final cost values achieved using various initial values in the optimization tool. $\mathbf{b}$ The number of iterations needed for the optimization tool to converge using various initial values. c The final cost values with respect to the numbers of iterations for different initial values. $\mathbf{d}$ The reliable initial guesses in the vicinity of the desired capacitor values that promised to converge to the global minimum
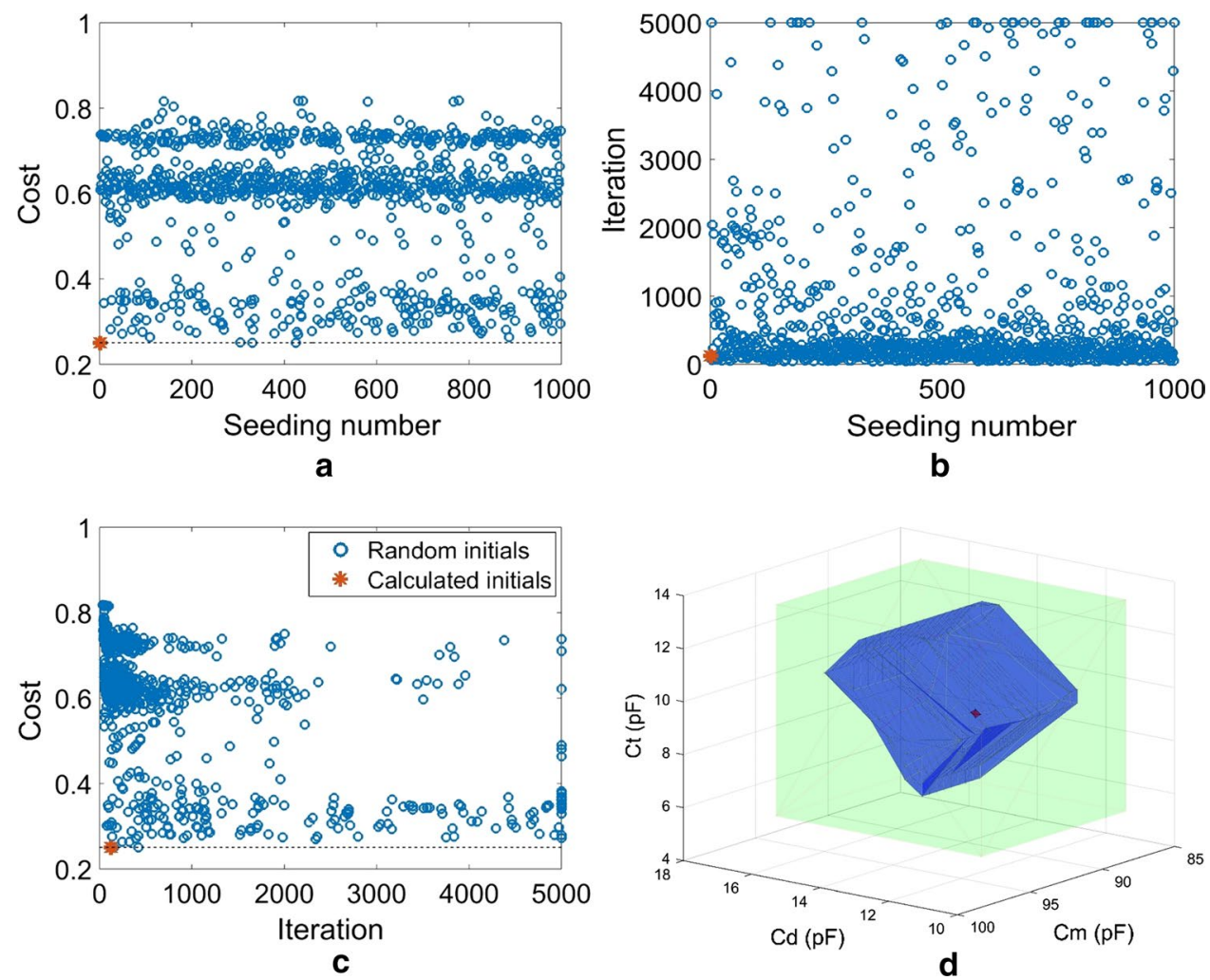
Table 1 Comparison between the design duration of the original co-simulation method and the proposed method

\begin{tabular}{|c|c|c|c|c|c|c|c|}
\hline & $\begin{array}{l}\text { Number of } \\
\text { independent } \\
\text { capacitors }\end{array}$ & $\begin{array}{l}\text { Analytic } \\
\text { calculations }\end{array}$ & $\begin{array}{l}\text { EM simulation } \\
\text { of the whole coil }\end{array}$ & $\begin{array}{l}\text { Circuit } \\
\text { simulation of } \\
\text { a single loop }\end{array}$ & $\begin{array}{l}\text { Interstage } \\
\text { optimization }\end{array}$ & $\begin{array}{l}\text { Final } \\
\text { optimization }\end{array}$ & Total \\
\hline \multicolumn{8}{|l|}{ Coil 1} \\
\hline \multicolumn{8}{|c|}{ Phantom } \\
\hline $\mathrm{O}$ & 3 & - & $40 \min$ & - & - & $13.5 \mathrm{~h}$ & $\approx 14 \mathrm{~h}$ \\
\hline M & 3 & $<1 \mathrm{~s}$ & $40 \min$ & $<1 \mathrm{~s}$ & $15 \mathrm{~s}$ & $15 \mathrm{~s}$ & $\approx 40 \mathrm{~min}$ \\
\hline \multicolumn{8}{|c|}{ Head } \\
\hline $\mathrm{O}$ & 17 & - & $35 \min$ & - & - & $46 \mathrm{~h}$ & $\approx 47 \mathrm{~h}$ \\
\hline M & 17 & $<1 \mathrm{~s}$ & $35 \min$ & $4 \mathrm{~s}$ & $90 \mathrm{~s}$ & $200 \mathrm{~s}$ & $\approx 40 \mathrm{~min}$ \\
\hline \multicolumn{8}{|l|}{ Coil 2} \\
\hline \multicolumn{8}{|c|}{ Phantom } \\
\hline $\mathrm{O}$ & 3 & - & $80 \mathrm{~min}$ & - & - & $18.5 \mathrm{~h}$ & $\approx 20 \mathrm{~h}$ \\
\hline M & 3 & $<1 \mathrm{~s}$ & $80 \min$ & $<1 \mathrm{~s}$ & $20 \mathrm{~s}$ & $25 \mathrm{~s}$ & $\approx 1.5 \mathrm{~h}$ \\
\hline \multicolumn{8}{|c|}{ Head } \\
\hline $\mathrm{O}$ & 25 & - & $115 \mathrm{~min}$ & - & - & $53 \mathrm{~h}$ & $\approx 55 \mathrm{~h}$ \\
\hline M & 25 & $<1 \mathrm{~s}$ & $115 \mathrm{~min}$ & $5 \mathrm{~s}$ & $3 \min$ & $300 \mathrm{~s}$ & $\approx 2 \mathrm{~h}$ \\
\hline \multicolumn{8}{|l|}{ Coil 3} \\
\hline \multicolumn{8}{|c|}{ Phantom } \\
\hline $\mathrm{O}$ & 3 & - & $140 \mathrm{~min}$ & - & - & $22.5 \mathrm{~h}$ & $\approx 25 \mathrm{~h}$ \\
\hline M & 3 & $<1 \mathrm{~s}$ & $140 \mathrm{~min}$ & $<1 \mathrm{~s}$ & $30 \mathrm{~s}$ & $30 \mathrm{~s}$ & $\approx 2.5 \mathrm{~h}$ \\
\hline \multicolumn{8}{|c|}{ Body } \\
\hline $\mathrm{O}$ & 33 & - & $220 \mathrm{~min}$ & - & - & $71 \mathrm{~h}$ & $\approx 74.5 \mathrm{~h}$ \\
\hline M & 33 & $<1 \mathrm{~s}$ & $220 \mathrm{~min}$ & $5 \mathrm{~s}$ & $5 \mathrm{~min}$ & $400 \mathrm{~s}$ & $\approx 4 \mathrm{~h}$ \\
\hline \multicolumn{8}{|l|}{ Coil 4} \\
\hline \multicolumn{8}{|c|}{ Phantom } \\
\hline $\mathrm{O}$ & 4 & - & $140 \mathrm{~min}$ & - & - & $26 \mathrm{~h}$ & $\approx 28.5 \mathrm{~h}$ \\
\hline M & 4 & $<1 \mathrm{~s}$ & $140 \mathrm{~min}$ & $<1 \mathrm{~s}$ & $40 \mathrm{~s}$ & $40 \mathrm{~s}$ & $\approx 3 \mathrm{~h}$ \\
\hline \multicolumn{8}{|c|}{ Head } \\
\hline $\mathrm{O}$ & 34 & - & $195 \min$ & - & - & $93.5 \mathrm{~h}$ & $\approx 97 \mathrm{~h}$ \\
\hline M & 34 & $<1 \mathrm{~s}$ & $195 \mathrm{~min}$ & $<1 \mathrm{~s}$ & $6 \min$ & $425 \mathrm{~s}$ & $\approx 3.5 \mathrm{~h}$ \\
\hline \multicolumn{8}{|l|}{ Coil 5} \\
\hline \multicolumn{8}{|c|}{ Phantom } \\
\hline $\mathrm{O}$ & 4 & - & $140 \mathrm{~min}$ & - & - & $25 \mathrm{~h}$ & $\approx 27.5 \mathrm{~h}$ \\
\hline M & 4 & $<1 \mathrm{~s}$ & $140 \mathrm{~min}$ & $<1 \mathrm{~s}$ & $40 \mathrm{~s}$ & $40 \mathrm{~s}$ & $\approx 2.5 \mathrm{~h}$ \\
\hline \multicolumn{8}{|l|}{ Head } \\
\hline $\mathrm{O}$ & 34 & - & $160 \mathrm{~min}$ & - & - & $97.5 \mathrm{~h}$ & $\approx 100 \mathrm{~h}$ \\
\hline M & 34 & $<1 \mathrm{~s}$ & $160 \mathrm{~min}$ & $<1 \mathrm{~s}$ & $6 \min$ & $400 \mathrm{~s}$ & $\approx 3 \mathrm{~h}$ \\
\hline \multicolumn{8}{|l|}{ Coil 6} \\
\hline \multicolumn{8}{|c|}{ Phantom } \\
\hline $\mathrm{O}$ & 12 & - & $240 \mathrm{~min}$ & - & - & $55 \mathrm{~h}$ & $\approx 59 \mathrm{~h}$ \\
\hline M & 12 & $<1 \mathrm{~s}$ & $240 \mathrm{~min}$ & $<1 \mathrm{~s}$ & $2 \min$ & $100 \mathrm{~s}$ & $\approx 4 \mathrm{~h}$ \\
\hline \multicolumn{8}{|c|}{ Body } \\
\hline $\mathrm{O}$ & 36 & - & $250 \mathrm{~min}$ & - & - & $174 \mathrm{~h}$ & $\approx 178 \mathrm{~h}$ \\
\hline M & 36 & $<1 \mathrm{~s}$ & $250 \mathrm{~min}$ & $<1 \mathrm{~s}$ & $6 \min$ & $450 \mathrm{~s}$ & $\approx 4.5 \mathrm{~h}$ \\
\hline
\end{tabular}

termination constraints of a maximum of 5000 iterations and a convergence tolerance of $10^{-4}$, was utilized.

As we argued, if the initial guess in a complex optimization problem is close to the global minimum, the problem can be solved using a simple optimization algorithm. In the case of the eight-channel DBC, to determine how close is close enough, the initial guesses for the three capacitor values are scanned between 4 to $14 \mathrm{pf}$ for $C_{\mathrm{t}}, 85 \mathrm{pf}$ to $100 \mathrm{pf}$ 
Table 2 Scattering parameters of the DBC. (a) Experimentally measured. (b) Obtained from the EM simulation

\begin{tabular}{llllllllll}
\hline & Channel & 1 & 2 & 3 & 4 & 5 & 6 & 7 & 8 \\
\hline Experiment & 1 & -28 & & & & & & \\
& 2 & -11 & -29 & & & & & \\
& 3 & -29 & -12 & -29 & & & & \\
& 4 & -21 & -29 & -11 & -30 & & & \\
& 5 & -19 & -20 & -30 & -11 & -28 & & & \\
& 6 & -20 & -18 & -20 & -29 & -12 & -29 & & \\
Simulation & 7 & -30 & -21 & -19 & -20 & -30 & -11 & -29 & \\
& 1 & -12 & -29 & -20 & -18 & -20 & -29 & -11 & -28 \\
& 2 & -30 & & & & & & & \\
& 3 & -9.6 & -30 & & & & & & \\
& 4 & -30 & -9.6 & -26 & & & & & \\
& 5 & -21 & -29 & -9.6 & -27 & & & & \\
& 6 & -19 & -21 & -29 & -9.6 & -28 & & \\
& 7 & -21 & -19 & -21 & -29 & -9.6 & -25 & & \\
& 8 & -30 & -21 & -19 & -21 & -29 & -9.6 & -32 & \\
& & -10 & -30 & -21 & -19 & -21 & -31 & -9.6 & -28 \\
\hline
\end{tabular}

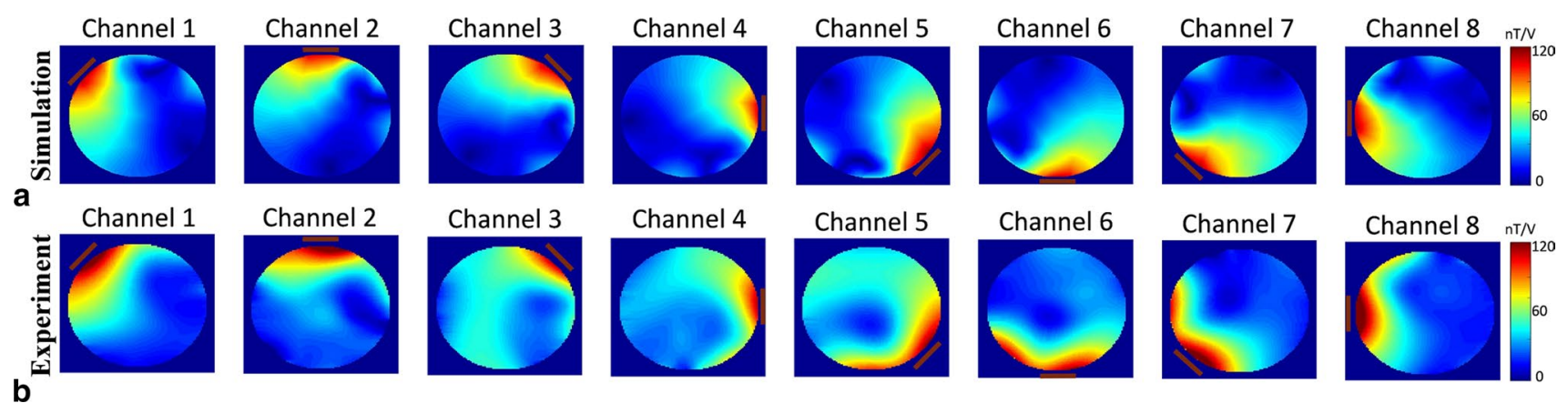

Fig. 8 a Simulation $\mathbf{b}$ and MRI experiment results for $\mathrm{B}_{1}{ }^{+}$-map of each channel

for $C_{\mathrm{m}}$, and $10 \mathrm{pf}$ to $18 \mathrm{pf}$ for $C_{\mathrm{d}}$. For this example-case, the initial capacitor values that fall into the volume shown in Fig. 7 d, the minimum value was obtained using the steepest descent algorithm is the global minimum. The red diamond shown in the figure represents the fine estimate of the initial capacitor values that is within this volume.

\section{DBC implementation}

The nonideal construction of the coil and tolerances of the capacitor values result in slight changes in the resonance frequency $(\sim 1 \mathrm{MHz})$ and matching. The capacitors, placed on the upper ring, were used for matching purposes $\left(C_{\mathrm{m}}\right)$ and the value of each of these capacitors was $90 \mathrm{pF}$. The variable capacitors on the lower ring were utilized as tuning capacitors $\left(C_{\mathrm{t}}\right)$ and trimmed between 7.5 and $9.5 \mathrm{pF}$ for fine-tuning. Finally, for decoupling purposes, $14.9 \mathrm{pF}$ capacitors were used on the rungs of the coil $\left(C_{\mathrm{d}}\right)$.
Table 2 shows reflection and coupling coefficients in $\mathrm{dB}$ for the DBC at 123.2 MHz. All numbers in Table 2a were obtained by bench-top measurement of the $S$-parameters of the constructed coil using the network analyzer. The maximum of the reflection coefficient $\left(S_{\mathrm{nn}}\right)$ was $-28 \mathrm{~dB}$. In the worst case, the mutual coupling coefficient $\left(S_{\mathrm{mn}}\right)$ for the adjacent channels was $-11 \mathrm{~dB}$. However, for nonadjacent channels, it was $-18 \mathrm{~dB}$. Table $2 \mathrm{~b}$ shows the $S$-parameters obtained in the EM simulation environment for the corresponding DBC.

The ratio of total reflected power to input power was $18 \%$ in the case of the constructed DBC but was $25 \%$ in the simulation environment. The difference between simulated and measured reflection values is possibly due to a mismatch between the geometry of the constructed coil and the simulation model. Besides, the capacitors were modeled as lossless in the EM simulations. The equivalent series resistance of the capacitors that we used may also play a role in this small error. 


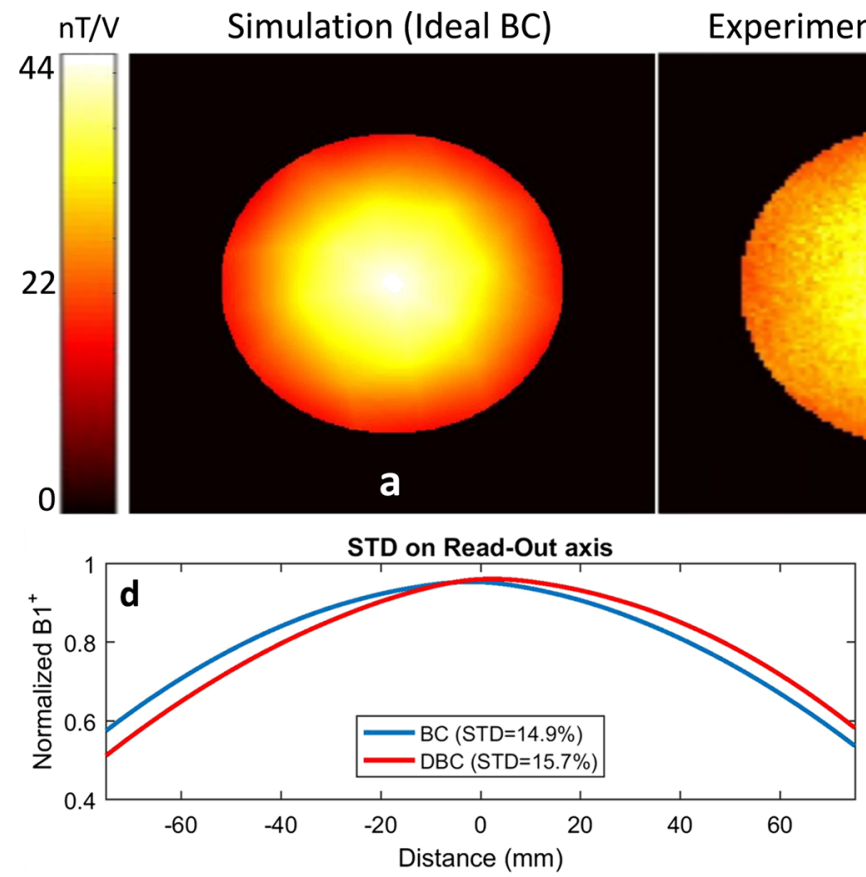

Fig. $9 \mathrm{a} \mathrm{B}_{1}{ }^{+}$-map of CP-mode of a standard $\mathrm{BC}$ that was achieved using the proper EM simulation. b CP mode that was generated using the constructed DBC. c The GRE image taken from a uniform phantom using the DBC. The sequence parameters are $\mathrm{TR}=100 \mathrm{~ms}$, $\mathrm{TE}=12 \mathrm{~ms}, \mathrm{NEX}=1,128 \times 128, \mathrm{FOV}=20 \mathrm{~cm}$, and slice thick-

\section{Field maps}

Once the $B_{I}{ }^{+}$maps are known, the TxArray system can be utilized for many applications, such as RF shimming [1-4]. The maps were acquired using the technique discussed in the Methods and Materials section. Figure 8 shows both the simulated and measured maps.

To provide proof of proper operation of the $\mathrm{DBC}$, we implemented RF shimming. To do so, we performed an EM simulation related to an ideal birdcage coil (BC) with the same size as the designed DBC. Quadrature excitation was used in the simulation environment to excite the circularly polarized (CP) mode of the BC (Fig. 9a). In the shimming process, we considered the $\mathrm{B}_{1}{ }^{+}$profile corresponding to the $\mathrm{CP}$ mode as the desired profile. We optimized the phase and magnitude for each channel using the steepest descent method. In this proof-of-operation study, we did not include the power criterion in the optimization problem. Panels a and $\mathrm{b}$ of Fig. 9 demonstrate the simulated and measured $\mathrm{B}_{1}^{+}$-maps for the desired CP mode and the optimized profile, respectively. Figure $9 \mathrm{c}$ is a gradient-echo MR image obtained using the optimized excitations.

The comparison between the homogeneity performance of the BC and DBC can be investigated using the standard deviation of the $B_{I}{ }^{+}$inside the phantom. Figure $9 \mathrm{~d}$ demonstrates the relative standard deviation of $B_{1}^{+}$on the readout axis for both the BC and DBC. Similarly, Fig. 9e shows the same parameter for both coils on the phase encoding axis. Accordingly, the relative standard deviation on the readout axis was $14.9 \%$ and $15.7 \%$ for the $\mathrm{BC}$ and $\mathrm{DBC}$, respectively. On the phase encoding axis, this was $15.2 \%$ and $13.8 \%$, respectively, for the BC and DBC. These results indicate that we managed to design $\mathrm{DBC}$ as desired, that is it has a mode of operation very similar to BC.

\section{Discussion}

In this paper, accelerating the co-simulation method for the design of TxArray coils is studied using equivalent circuit models. In the original co-simulation method proposed by Kozlov et al. [38], an EM simulation of the coil in the presence of an imaging object is performed while all lumped elements are replaced by excitation ports. Afterward, the resultant $S$-parameters are imported to a circuit simulator and a time-consuming optimization is performed on the lumped element values, due to the excessive number of local minima in the problem. In the proposed method, we show that starting from proper initial guesses for the capacitor values in the optimization process helps in quickly finding the optimum 
capacitor values. As an example, an eight-channel headdegenerate birdcage coil is constructed using the obtained values, during which the design process is accelerated by a factor of 20.

As shown in Table 1, the acceleration occurs in the final optimization stage of the design process. Correspondingly, the acceleration factor mostly depends on the number of unknown parameters (capacitor values) during the optimization. In the case of the coils in Fig. 4 in the presence of the cylindrical phantom, the number of independent capacitors was decreased to three, three, three, four, four, and 12, respectively, due to the cylindrical symmetry. However, in the case of the loading with the human model, these numbers were $17,25,33,34,34$, and 36 , respectively. Note that, to assign the independent optimization parameter to the decoupling capacitors, we only considered the geometrical features of the coils regardless of the loading schemes.

Currently, degenerate birdcage coils are not used extensively in ultra-high field applications. However, these coils are one of the best choices for a $3 \mathrm{~T}$ transmit array coil because it has a mode of operation similar to a conventional birdcage coil. Unfortunately, the complexity of their design is a disadvantage. This manuscript partially addresses this problem, as well. Although we presented the circuit model for DBCs and used the corresponding circuit models for five other types of TxArray coils in this work, the proposed method can be easily extended and utilized in the design of other types of TxArray coils, as well, using a proper equivalent circuit model for the intended coil. The corresponding MATLAB scripts for the presented examples (i.e., six different coils with two different loading schemes) which include a relatively large variety of TxArrays to prove the concept are available online as the supplementary material.

It should be noted that in the presented equivalent circuit model, the inductors represent the copper strips of the coil. However, this representation is only valid if the wavelength is much greater than the size of the strips. As the length of the wire increases, a multistage RLC network or even more complex models may be necessary to model a single conductive strip [51].

Furthermore, determining the optimum geometry of the TxArray coils in terms of either their dimensions or their shape is always demanding. Since tuning and decoupling of a candidate geometry is usually a time-consuming process, it is difficult to explore the many varieties of possible geometries. The proposed method can shorten the design process and determine the feasibility of a design, allowing a greater number of candidate designs to be examined.

The workstation used in this study is a mediocre system with enhanced memory. Clearly, more powerful systems can make the design process faster. Additionally, the gradient descent method we used as an optimization algorithm may not be the best choice for this problem. There are many sophisticated minimization algorithms for problems involving multiple local minima. As can be understood from Fig. 7, this problem has many local minima. Here, instead of optimizing the optimization algorithm, we chose to work on the minimization of the search interval. Our proposed solution should also help all other optimization algorithms since the initial guess is close to the optimum point.

In the construction of the coil, fine adjustment of the tuning capacitors was necessary. We believe this is mainly due to tolerances in the capacitors and imperfections in their construction. These can be handled by measuring the capacitance errors and 3D printing the coil-housing. Some additional errors are due to the errors of the EM simulations. To reduce these errors, convergence criteria can be reduced, though this significantly increases the simulation time and the memory requirement. However, the errors due to EM simulation are significantly fewer in number than those due to other sources of error.

Acknowledgements This project was supported by ASELSAN A.S. Ankara, Turkey. MREPT experiments were performed by the aid of Mr. Safa Ozdemir using the facilities of UMRAM, Bilkent University, Ankara, Turkey.

Author contributions AST: responsible for study conception and design, acquisition of data, analysis and interpretation of data, and drafting of manuscript. EK: involved in study conception and design. UG: advised and developed methods for the acquisition of data. SE: advised and developed methods to analyze the data. EA: responsible for study conception and design, analysis and interpretation of data, drafting of the manuscript, and critical revision of the manuscript.

\section{Compliance with ethical standards}

Conflict of interest The authors declare that they have received a research grant from ASELSAN.

Ethical approval The editorial does not contain any studies with human participants or animals performed by any of the authors.

Human and animal rights This article does not contain any studies with human participants or animals performed by any of the authors.

\section{References}

1. Tropp J (2004) Image brightening in samples of high dielectric constant. J Magn Reson 167(1):12-24

2. Bernstein MA, Huston J, Ward HA (2006) Imaging artifacts at 3.0 T. J Magn Reson Imaging 24(4):735-746

3. Childs AS, Malik SJ, O'Regan DP, Hajnal JV (2013) Impact of number of channels on RF shimming at 3T. Magn Reson Mater Biol Phy Med 26(4):401-410

4. Setsompop K, Wald LL, Alagappan V, Gagoski B, Hebrank F, Fontius U, Schmitt F, Adalsteinsson E (2006) Parallel RF transmission with eight channels at 3 Tesla. Magn Reson Med 56(5):1163-1171 
5. Guérin B, Gebhardt M, Cauley S, Adalsteinsson E, Wald LL (2014) Local specific absorption rate (SAR), global SAR, transmitter power, and excitation accuracy trade-offs in low flip-angle parallel transmit pulse design. Magn Reson Med 71(4):1446-1457

6. Cloos MA, Luong M, Ferrand G, Amadon A, Le Bihan D, Boulant $\mathrm{N}$ (2010) Local SAR reduction in parallel excitation based on channel-dependent Tikhonov parameters. J Magn Reson Imaging 32(5):1209-1216

7. Zhu Y, Alon L, Deniz CM, Brown R, Sodickson DK (2012) System and SAR characterization in parallel RF transmission. Magn Reson Med 67(5):1367-1378

8. van den Bergen B, Van den Berg CA, Bartels LW, Lagendijk JJ (2007) 7 T body MRI: B1 shimming with simultaneous SAR reduction. Phys Med Biol 52(17):5429

9. Guérin B, Gebhardt M, Serano P, Adalsteinsson E, Hamm M, Pfeuffer J, Nistler J, Wald LL (2015) Comparison of simulated parallel transmit body arrays at $3 \mathrm{~T}$ using excitation uniformity, global SAR, local SAR, and power efficiency metrics. Magn Reson Med 73(3): 1137-1150

10. Lattanzi R, Sodickson DK, Grant AK, Zhu Y (2009) Electrodynamic constraints on homogeneity and radiofrequency power deposition in multiple coil excitations. Magn Reson Med 61(2):315-334

11. Sadeghi-Tarakameh A, Torrado-Carvajal A, Ariyurek C, Atalar E, Adriany G, Metzger G, Lagore R, DelaBarre L, Grant A, Van de Moortele P, Ugurbil K, Eryaman Y (2018) Optimizing the topography of transmit coils for SAR management. In: Proceeding of the 26th Joint Annual Meeting ISMRM-ESMRMB, Paris, France. p 0297

12. Sadeghi-Tarakameh A, DelaBarre L, Lagore RL, Torrado-Carvajal A, Wu X, Grant A, Adriany G, Metzger GJ, Van de Moortele PF, Ugurbil K, Atalar E, Eryaman Y (2020) In vivo human head MRI at $10.5 \mathrm{~T}$ : A radiofrequency safety study and preliminary imaging results. Magn Reson Med 84(1):484-496

13. Sadeghi-Tarakameh A, Wu X, Ulus F, Eryaman Y, Atalar E (2019) Ultimate Intrinsic Specific Absorption Rate Efficiency. In: Proceedings of the 27th Annual Meeting of ISMRM, Montreal, QC, Canada. p 4166

14. Katscher U, Börnert P (2006) Parallel RF transmission in MRI. NMR Biomed 19(3):393-400

15. Zhu Y (2004) Parallel excitation with an array of transmit coils. Magn Reson Med 51(4):775-784

16. Pauly J, Nishimura D, Macovski A (1989) A linear class of largetip-angle selective excitation pulses. J Magn Reson 82(3):571-587

17. Hardy CJ, Cline HE (1989) Spatial localization in two dimensions using NMR designer pulses. J Magn Reson 82(3):647-654

18. Eryaman Y, Akin B, Atalar E (2011) Reduction of implant RF heating through modification of transmit coil electric field. Magn Reson Med 65(5):1305-1313

19. Eryaman Y, Guerin B, Akgun C, Herraiz JL, Martin A, Torrado-Carvajal A, Malpica N, Hernandez-Tamames JA, Schiavi E, Adalsteinsson E (2015) Parallel transmit pulse design for patients with deep brain stimulation implants. Magn Reson Med 73(5):1896-1903

20. Eryaman Y, Turk EA, Oto C, Algin O, Atalar E (2013) Reduction of the radiofrequency heating of metallic devices using a dualdrive birdcage coil. Magn Reson Med 69(3):845-852

21. Kazemivalipour E, Keil B, Vali A, Rajan S, Elahi B, Atalar E, Wald LL, Rosenow J, Pilitsis J, Golestanirad L (2019) Reconfigurable MRI technology for low-SAR imaging of deep brain stimulation at 3T: application in bilateral leads, fully-implanted systems, and surgically modified lead trajectories. NeuroImage 199:18-29

22. Golestanirad L, Kazemivalipour E, Keil B, Downs S, Kirsch J, Elahi B, Pilitsis J, Wald LL (2019) Reconfigurable MRI coil technology can substantially reduce RF heating of deep brain stimulation implants: first in-vitro study of RF heating reduction in bilateral DBS leads at 1.5 T. PLoS ONE 14:8

23. Lee RF, Hardy CJ, Sodickson DK, Bottomley PA (2004) Lumpedelement planar strip array (LPSA) for parallel MRI. Magn Reson Med 51(1):172-183

24. Adriany G, de Moortele V, Wiesinger F, Moeller S, Strupp JP, Andersen P, Snyder C, Zhang X, Chen W, Pruessmann KP (2005) Transmit and receive transmission line arrays for 7 Tesla parallel imaging. Magn Reson Med 53(2):434-445

25. Roemer PB, Edelstein WA, Hayes CE, Souza SP, Mueller O (1990) The NMR phased array. Magn Reson Med 16(2):192-225

26. Wu B, Qu P, Wang C, Yuan J, Shen GX (2007) Interconnecting $\mathrm{L} / \mathrm{C}$ components for decoupling and its application to low-field open MRI array. Conc Magn Reson Part B Magn Reson Eng 31(2):116-126

27. Raaijmakers AJE, Ipek O, Klomp DWJ, Possanzini C, Harvey PR, Lagendijk JJW, van Den Berg CAT (2011) Design of a radiative surface coil array element at $7 \mathrm{~T}$ : the single-side adapted dipole antenna. Magn Reson Med 66(5):1488-1497

28. Raaijmakers AJE, Italiaander M, Voogt IJ, Luijten PR, Hoogduin JM, Klomp DWJ, van Den Berg CAT (2016) The fractionated dipole antenna: a new antenna for body imaging at $7 \mathrm{~T}$ esla. Magn Reson Med 75(3):1366-1374

29. Steensma B, Andrade A, Klomp D, Van den Berg C, Luijten P, Raaijmakers (2016) A Body imaging at 7 Tesla with much lower SAR levels: an introduction of the Snake Antenna array. In: Proceedings of the 24th Annual Meeting of ISMRM, Singapore. p 0395

30. Ertürk MA, Wu X, Eryaman Y, Van de Moortele PF, Auerbach EJ, Lagore RL, DelaBarre L, Vaughan JT, Uğurbil K, Adriany G (2017) Toward imaging the body at 10.5 tesla. Magn Reson Med 77(1):434-443

31. Sadeghi-Tarakameh A, Adriany G, Metzger GJ, Lagore RL, Jungst S, DelaBarre L, Van de Moortele PF, Ugurbil K, Atalar E, Eryaman Y (2020) Improving radiofrequency power and specific absorption rate management with bumped transmit elements in ultra-high field MRI. Magn Reson Med. https://doi.org/10.1002/ mrm. 28382

32. Sadeghi-Tarakameh A, Jungst $\mathrm{S}$, Wu X, Lanagan M, Adriany G, Metzger GJ, Van de Moortele P-F, Ugurbil K, Atalar E, Eryaman Y (2019) A New Coil Element for Highly-Dense Transmit Arrays: An Introduction to Non-Uniform Dielectric Substrate (NODES) Antenna. In: Proceedings of the 27th Annual Meeting of ISMRM, Montreal, QC, Canada. p 0732

33. Sadeghi-Tarakameh A, Torrado-Carvajal A, Lagore RL, Moen S, Wu X, Adriany G, Metzger GJ, DelaBarre L, Ugurbil K, Atalar E, Eryaman Y (2019) Toward Human Head Imaging at 10.5 T Using an Eight-Channel Transmit/Receive Array of Bumped Fractionated Dipoles. In: Proceedings of the 27th Annual Meeting of ISMRM, Montreal, QC, Canada. p 0430

34. Alagappan V, Nistler J, Adalsteinsson E, Setsompop K, Fontius U, Zelinski A, Vester M, Wiggins GC, Hebrank F, Renz W (2007) Degenerate mode band-pass birdcage coil for accelerated parallel excitation. Magn Reson Med 57(6):1148-1158

35. Stara R, Tiberi G, Morsani F, Symms M, Fantacci ME, Marletta M, Zampa V, Pendse M, Retico A, Rutt BK (2017) A degenerate birdcage with integrated $\mathrm{Tx} / \mathrm{Rx}$ switches and butler matrix for the human limbs at 7 T. Appl Magn Reson 48(3):307-326

36. Sadeghi-Tarakameh A, Kazemivalipour E, Demir T, Gundogdu U, Atalar E (2018) Accelerating the Co-Simulation Method for Fast Design of Transmit Array Coils: An Example Study on a Degenerate Birdcage Coil. In: Proceeding of the 26th Joint Annual Meeting ISMRM-ESMRMB, Paris, France. p 4404

37. Kazemivalipour E, Sadeghi Tarakameh A, Yilmaz U, Acikel V, Sen B, Atalar E (2018) A 12-channel degenerate birdcage body transmit array coil for 1.5T MRI scanners. In: Proceeding of the 
26th Joint Annual Meeting ISMRM-ESMRMB, Paris, France. p 1708

38. Kozlov M, Turner R (2009) Fast MRI coil analysis based on 3-D electromagnetic and RF circuit co-simulation. J Magn Reson 200(1):147-152

39. Kozlov M, Turner R Analysis of RF transmit performance for a multi-row multi-channel MRI loop array at 300 and $400 \mathrm{MHz}$. In: Microwave Conference Proceedings (APMC), 2011 Asia-Pacific, 2011. IEEE, pp 1190-1193

40. Kozlov M, Turner R Analysis of RF transmit performance for a 7T dual row multichannel MRI loop array. In: Engineering in Medicine and Biology Society, EMBC, 2011 Annual International Conference of the IEEE, 2011. IEEE, pp 547-553

41. Cheng DK (1993) Fundamentals of engineering electromagnetics. Pearson Education, New Jersey, pp 136-142

42. Grover FW (2004) Inductance calculations: working formulas and tables. Dover Publication, New York, pp 20-76

43. Tarakameh AS (2016) Design of a birdcage-like radio frequency transmit array coil for the magnetic resonance imaging using equivalent circuit model. Bilkent University, Thesis

44. Jin J (1998) Electromagnetic analysis and design in magnetic resonance imaging, vol 1. CRC Press, Florida

45. Kozlov M, Turner R (2010) A comparison of Ansoft HFSS and CST microwave studio simulation software for multi-channel coil design and SAR estimation at 7T MRI. Piers Online 6(4):395-399

46. Pozar DM (2009) Microwave engineering, 2nd edn. John Wiley \& Sons, New York, pp 182-244
47. Habashy TM, Abubakar A (2004) A general framework for constraint minimization for the inversion of electromagnetic measurements. Progr Electromagn Res 46:265-312

48. Etminan A, Sadeghi A, Gurel L (2014) Electromagnetic imaging of three-dimensional dielectric objects with Newton minimization. In: Antennas and Propagation Society International Symposium (APS-URSI), IEEE, p 876-877

49. Hayes CE, Eash MG (1987) Shield for decoupling RF and gradient coils in an NMR apparatus. Google Patents

50. Roemer PB, Edelstein WA (1989) RF shield for RF coil contained within gradient coils of NMR imaging device. Google Patents

51. Bahl IJ (2003) Lumped elements for RF and microwave circuits. Artech house, Boston

52. Hafalir FS, Oran OF, Gurler N, Ider YZ (2014) Convectionreaction equation based magnetic resonance electrical properties tomography (cr-MREPT). IEEE Trans Med Imaging 33(3):777-793

53. Gurler N, Ider YZ (2017) Gradient-based electrical conductivity imaging using MR phase. Magn Reson Med 77(1):137-150

54. Sacolick LI, Wiesinger F, Hancu I, Vogel MW (2010) B1 mapping by Bloch-Siegert shift. Magn Reson Med 63(5):1315-1322

55. Turk EA, Ider YZ, Ergun AS, Atalar E (2015) Approximate Fourier domain expression for Bloch-Siegert shift. Magn Reson Med 73(1):117-125

Publisher's Note Springer Nature remains neutral with regard to jurisdictional claims in published maps and institutional affiliations. 